\title{
CONTENTS
}

The Diagnosis of Some Acute Eruptions. By Arthur Whitfield, M.D., F.R.C.P. •

$\therefore 145$

The Prophylaxis of Scarlet Fever and DiPHTHERIA.

By David C. Krrkhope, M.D., D.P.H.

Poliomyelitis and Polio-Encephalitis.

By E. A. Cockayne, M.D., F.R.C.P.

Functional Nervous Disorders of the Heart.

By R. Travers SmIth, M.D., F.R.C.P.-

(Concluded)
Post-Graduate Clinical Demonstration at the Hampstead General Hospital.

By Adolphe Abrahams, M.D. 155

EDITORIAL NOTES.

Post-Graduate Work in the Dominions $\quad \cdot 158$

The Need for Insurance by Doctors • • • 159

OfFictal Intmations . $\quad . \quad . \quad . \quad . \quad .160$

Fellowship of Medicine and Post-Graduate Medical Association.-Officers, 1927 . • 5

SPecial Courses $\quad$. $\quad$. $\quad$.

\section{THE DIAGNOSIS OF SOME ACUTE ERUPTIONS.*}

\author{
BY
}

\author{
ARTHUR WHITFIELD, M.D., F.R.C.P. LoND., \\ LATE PHYGICLAN TO THE gKIN DHPARTMENT, KING'S \\ COLLEGE HOSPITAL.
}

I sHoULD like, first, to offer you a few words on the use of the word diagnosis. It appears to me that it is used in two senses, one indicating the nature of the disease, and the other merely the condition observed. To make this clear I give two instances. If $I$ say that an eruption is syphilitic I give you in a word the nature and ætiology of the disease, but if $I$ say that an eruption is eczematous I merely refer to the clinical and anatomical peculiarities which, in my opinion, may be called forth by causes so different as the local contact with a poisonous plant-e.g., Primula obconicaor the ingestion of unsuitable food. I shall, therefore, endeavour to indicate to you as far as possible the cause that is to be sought in cases of the second class.

I begin, then, with the generalised erythemata. They are commonly divided into the scarlatiniform and the morbilliform. In the former type the diagnosis from scarlatina is obviously the important point. That this can be made in every case I do not assert, but $I$ think that in the vast majority it is possible, and I will try to indicate the signs for which I look.

\section{Type and Distribution of the Eruption.}

First, as to the type of the eruption. In both cases, of course, this is almost purely erythematous, that is, there is little or no elevation or disturb-

*A Fellowship of Medicine Lecture delivered on March 3rd, ance of the skin surface. Careful examination, however, often reveals the fact that in true scarlatina the individual lesion is more distinctly punctiform, whereas in the scarlatiniform erythema the rash is a more diffuse and even redness. The best place to look for this is, I think, the proximal phalanx of the forefinger, where the rash of scarlatina may almost be described as a minute follicular papule.

Secondly, in scarlatina proper, if one looks at the flexures of the elbows and knees, one usually finds a curious smoky brown discolouration of the skin, and when it is present I consider this of considerable diagnostic importance. I have fairly often found it absent in scarlatina, but $I$ do not remember finding it present in the non-infectious erythema.

Thirdly, as regards distribution. About the localisation there is, I think, not much that is distinctive, but the scarlatiniform erythema may be said to be much more apt to be partial and to show rather sharply defined lines of demarcation between the erythematous and normal skin, and in some cases this is so marked as to be of considerable service.

One other point about the cutaneous appearances may be mentioned, and that is the relationship of the desquamation to the erythema. It is, of course, well known that marked desquamation may occur in Erythema scarlatiniforme as well as in scarlatina. It is, however, true to say that in scarlatina the rash, which is usually of short duration, has almost invariably faded before the beginning of the desquamation. In scarlatina desquamation of the face may begin as early as the fourth day after the appearance of the eruption, but in that case the eruption itself has usually faded in 24 to 48 hours-i.e., at least two days before the beginning of desquamation. In $E$. scarlatiniforme, on the other hand, it is the rule for desquamation to begin while the erythema 
is still manifest. This observation is one stressed a good deal by some French authors, and is one which my experience leads me to believe is correct.

I am not dealing with the temperature, the malaise, the tongue, the throat, or the onset of the two diseases, as these have been already dealt with by a previous lecturer, and, moreover, they may all be very equivocal at times; but I would draw your attention especially to the pulse, which shortly after the onset has in scarlatina a very characteristic bounding quality which I have not observed in $E$. scarlatiniforme, though I have always sought for it.

Lastly, in the matter of diagnosis the history of one or more previous attacks may be of service, since $E$. scarlatiniforme is a typically recurrent disease, though, of course, there must be a first attack.

\section{Cause of the Condition.}

The cause of $E$. scarlatiniforme is known only in some instances, and in those cases in which it recurs periodically $I$ have never been able to trace it. The commonest known causes are septic absorption from a suppurating wound, and eruptions due to the absorption of drugs, and with regard to the latter I would draw your attention to the fact that in patients with a marked idiosyncrasy, infinitesimal doses are sufficient. One case has been recorded in which the patient had a severe erythematous eruption with constitutional disturbance, owing to the fact that the dispenser had not washed out the measure which he had previously used for a quinine mixture prescribed for another patient.

The quinine erythema is for me the most difficult to distinguish from scarlatina, as it is frequently associated with intense constitutional disturbance, rigor, high temperature, and angina. The other drugs chiefly responsible are belladonna, the balsams, antipyrin, veronal, mercury, iodoform, and iodine, the last three usually by absorption from external applications.

In the morbilliform erythemata the diagnosis from the corresponding acute specific fevers, measles, and rubella, especially from the former, is usually easier to establish. In true measles the intense coryza and conjunctivitis are so characteristic, and in rubella the lymphatic nodes over the occiput are helpful. It is, however, as well in the presence of the occipital lymph nodes to search the scalp so as to eliminate other causes of lymphatic adenitis which may produce the coincidental occurrence of adenitis with an erythematous eruption. The possibility of enema rash must never, of course, be forgotten, and eruptions from unsuitable food or drugs must also be borne in mind.

\section{Uncommon Cases.}

I may here allude to a peculiar form of erythemato-urticarial eruption which I described some years ago occurring in patients who have suffered from an aseptic tissue destruction. The first case that I saw was that of a young lady whof received a severe blow on the shin from a cricket

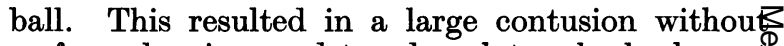
surface abrasion, and ten days later she broke out in an acute erythemato-urticarial eruption alfover the trunk and limbs, accompanied by severe pruritus. It is perhaps worthy of note that the eruption did not spread from the site of the injury ${ }_{\overline{0}}^{\circ}$ but flashed out with a general distribution from the first. Since then I have seen a few more cases from falls, sprains, and fractures, but they are curiously uncommon. To establish the realityo of this occurrence, I may say, first, that since my description cases of the same kind have beenrecognised by Prof. Rasch in Copenhagen; and $\overrightarrow{\mathrm{N}}$ secondly, that they have also been observed its France after the therapeutic injection of the patient's own blood-so-called auto-hæmotherapy

I have thought that the irritation has been diminished and the eruption cut short by the internal administration of turpentine, of course after a previous examination of the urine.

\section{Urticaria and Exudative Erythema.}

I may now pass on to those eruptions in which the texture of the skin is disturbed. These include् urticaria and the exudative types of erythema: certain vesicular and bullous diseases, and acute desquamating eruptions. The lesions of urticarias are so characteristic that the disease does not often present any difficulty in diagnosis unlesฐ in the distinction from some of the less well-defined forms of exudative erythema. In that case th $\vec{E}$ differential diagnosis is not of great importance as there is really hardly any sharp line of division? between them. The nodular forms of exudative. erythema are more important, since they are not infrequently mistaken for secondary syphilis. In. both eruptions the patient is liable to complain of general malaise and headache, in both the evening temperature may be raised a degree oo so, and, more confusing still, the mucous membrane of the mouth may be affected in both.

The points of distinction with regard to the eruption itself are the following:-The colour of. erythema multiforme is a very bright light-redis which turns in the older lesions to cyanotic blue? The colour of the secondary syphilide in its earlys stage is a salmon-pink, and in the later stage it usually turns to a brownish-red rather than blue The periphery of the erythema papule is sharplyo defined, and is sometimes surrounded by a rings of skin which is obviously paler than the normal? whereas the syphilitic papule has a rather ill-definedo edge and is usually surrounded by a somewha? reddish border. If of any size the erythema papul@ shows almost invariably a dark purple or almos: black centre, about a millimetre in diameterg and fading with difficulty or not at all on pressure, and I have never seen this in the syphilide. 
To the palpating finger both offer a sense of resistance, but whereas the erythema papule gives the sensation of fluid under high tension, the syphilide gives a sensation of toughness not unlike that of indiarubber. I must confess that to make this distinction requires a highly educated finger. Lastly, there are marked differences of localisation, since the sites of predilection in erythema multiforme are chiefly the backs of the hands and feet, the points of the elbows and knees, the cheeks and the ears, whereas the early rash of secondary syphilis is found more profusely on the front and back of the trunk, the forehead, the circumoral region of the face and the upper parts of the limbs. Nevertheless, I counsel you to be chary of attaching too much weight to localisation, as I regard it as an unreliable sign. I may mention here another disease of only moderately frequent occurrence, and consequently liable to give trouble in diagnosis -namely, pityriasis rosea. This commonly begins with a single patch on the trunk, the "herald patch," which may persist for from four to ten days before the outbreak of the generalised eruption, but this " herald patch" may not be present. The general eruption is often mistaken for secondary syphilis, as in some instances itching is entirely absent, though I should class it among the pruritic diseases. When seen fully out the eruption is somewhat multiform. There are raised areas of about $\frac{1}{4}$ to $\frac{1}{2}$ inch in diameter, scattered chiefly over the upper part of the trunk and arms on both extensor and flexor surfaces. Between these there are generally large numbers of small papules about a millimetre in diameter. In addition, the larger areas are usually oval in shape, with the long diameters lying in the lines of cleavage of the skin. The colour is of a bright salmon pink, and the lesions are raised and palpable. We have thus four similarities to the secondary syphilidenamely, multiformity, colour, arrangement on the lines of cleavage, and distribution. The points of distinction are that in pityriasis rosea the lesion is obviously œdematous rather than infiltrative in character, and that quite early there is a very characteristic form of scaling. This scaling in its earliest form consists of a pin's-head sized raising of the horny layer by means of an air vesicle. Microscopically $I$ believe there is a serous vesicle found in the depth of the epidermis, but the fluid evidently disappears before the horny layer is reached, since this never shows a serous vesicle beneath it. This air vesicle breaks and leaves a frayed edge of scaling, the free edge of which is turned towards the centre of the lesion. The scaling does not extend to the edge of the red œedematous papule, so that a very characteristic appearance results. The health is, of course, not usually disturbed.

Another eruption which may be confused with secondary syphilis is acute lichen planus. This, again, is usually an intensely pruritic disease, but I have seen the most acute cases in which itching was entirely absent. Here, again, we have a series of qualities which are common to lichen and to syphilis. Both may be generalised eruptions, both are infiltrative diseases, and in both the buccal mucous membrane is commonly affected. The points of distinction on which I rely are these : Taking, first, the individual lesion, that of lichen is seldom if ever round, but is triangular or polygonal when closely examined, that of syphilis is round; that of lichen is flat-topped from the beginning, while that of syphilis is acuminate until it begins to resolve ; that of lichen is situated chiefly around the sweat gland, and frequently shows a central depression corresponding with the sweat pore, while that of syphilis is chiefly follicular and, commonly shows a slight follicular horn or sometimes an indolent pustule. In most cases of lichen planus small aggregations of papules will be found which, when examined with a lens, show on their bluish-pink surface fine white stipplings, the socalled striæ of Wickham, and I have never seen these in any other disease. Lastly, the grouping of the lesions is of great significance. In lichen the papules are arranged on the limbs in short irregular lines which have been likened to the pattern that trickling raindrops make on a windowpane, and on the abdomen and chest they are generally arranged in short, more or less horizontal, lines, which really correspond with the anatomical arrangement of the sweat glands, whereas in syphilis the lesions are grouped in discs about an inch in diameter, the centre of the disc being more intensely affected than the periphery.

\section{Erysipelas and Eczema.}

I pass now to the diagnosis of two diseases which partake of the œdematous and of the vesicobullous types, and are perhaps the most frequent source of confusion of any. I allude to erysipelas and acute eczema of the face.

In the first attack of acute erysipelas this is a mistake which I think should not arise. As far as I can see the only points of resemblance are that both are acute cedematous eruptions which tend to close the eyes. We can put the points of distinction clearly and fairly briefly. Erysipelas begins at one point from which it extends rapidly ; eczema nearly always flashes out over a large area at once; erysipelas is of very firm consistency, most marked at the edge which is abrupt and well defined; eczema is soft in consistency, the intensity of the eruption is greatest at its centre, and the edge fades away imperceptibly into the normal skin; in erysipelas the main surface is smooth and shining, whereas in eczema with a side light one can see fine miliary papules closely aggregated over the area; bullæ when present in erysipelas arise as such, whereas in eczema they are formed by the confluence of small vesicles, and bear on their surface the markings corresponding to the original vesicles. Last, but by no means least in importance, in erysipelas the temperature is raised several degrees, and the patient is severely ill, whereas the 
rise of temperature in acute eczema, if present at all, is only trifling, possibly a degree, and the patient who looks so ill feels quite well, though perhaps tormented with itching.

There is, however, another, the relapsing, form of erysipelas, to which I have given the descriptive name of relapsing streptococcal lymphangitis. This is in reality a chronic disease with acute exacerbations. The patient will generally give a history somewhat of this sort. At first there was an ordinary attack of acute erysipelas, with fever and illness, and this got well. After a variable period a second attack ensued over the same area, accompanied by less serious constitutional symptoms, and when it disappeared perhaps some slight thickening of the affected parts was left behind. Later, many attacks occurred, the intervening periods of health becoming progressively shorter and all constitutional symptoms remaining absent.

In these cases the sharp margin and defined outline are lost, bullæ do not arise, but the whole eruption is limited to a somewhat red and shining œdema with some permanent thickening. The

\section{THE PROPHYLAXIS OF SCARLET FEVER AND DIPHTHERIA.*}

BY

\author{
DAVID C. KIRKHOPE, M.D., D.P.H.,
} MEDICAL OFFICER OF HEALTH FOR TOTTENHAM, N.

I HAVE chosen this subject for consideration because I have come to the conclusion that we are on the eve of a revolution respecting the methods by which these diseases will be controlled.

If it was ever anticipated that isolation of the infectious sick in infectious diseases hospitals would result in the suppression of the maladies concerned, I think it will be conceded generally that those hopes have not been realised. Not only is this so, but there has been the disadvantage in these hospitals of cross infections with accompanying complications and mortality. These disadvantages are not negligible, for, quoting from the report of the Metropolitan Asylums Board for the year 1922 (the last year for which similar information is available), it is shown that 15,279 scarlet fever cases were admitted to the Metropolitan Asylums Board's hospitals and of these-

$\begin{array}{rrrr}1826 & \text { contracted one other disease, } \\ 118 & \text {, } & \text { two , diseases, } \\ 6 & \text { " } & \text { three , } & , \\ 2 & \text {, } & \text { four , } & ,\end{array}$

i.e., $12 \cdot 77$ per cent. of the cases contracted at

* A Lecture delivered before the North-East London PostGraduate College on Jan. 21 st, 1927. diagnosis is made on the permanent thickening, की the absence of the true eczematous signs, and the history of frequent relapse with serious fever and $\cong$ illness at the first one or two attacks. Such $\frac{\mathbb{\Phi}}{3}$ patients have nearly always a fissure somewhere in the vestibule of the nose, most commonly either ${ }^{\complement}$ at the anterior angle of the nasal orifice or at the $\overrightarrow{\vec{s}}$ junction of the ala nasi with the cheek. This healsfrom time to time, generally remaining slightly등 red, especially in cold weather. On the patient $\bar{c}$. developing a coryza it opens again and the strepto- $\widehat{\Phi}$ coccal lymphangitis recurs. Constitutional immunity has developed so far that the patient no longeres feels very ill, though perhaps slightly out of sorts, $\overrightarrow{0}$ and the main discomfort remains local. I regardthis as a very interesting condition, because it is $\bar{\sigma}_{\sigma}$ so good an example of the importance of generato constitutional immunity as contrasted with local tissue immunity. I have worked at it carefully. by means of attempted cultivation and inoculation, N but have been unsuccessful, and this is perhaps not surprising in view of the comparatively high state of constitutional resistance which is present.응

(To be concluded.)

least one other disease, and 48 deaths occurred amongst them.

Similarly with regard to diphtheria, 11,737 cases were admitted in 1922 , of whom 1207 contracted other diseases-viz., 10.28 per cent. Of these, 1136 contracted one other disease, 68 contracted two other diseases, 3 contracted three other diseases, and of these 79 died.

In the four years 1922-25 inclusive, 4967 cases were admitted under a wrong diagnosis of scarletō fever and 72 of them died, and in the same period ${ }^{3}$ 8634 wrongly diagnosed as diphtheria were admitted with 198 deaths.

\section{Scarlet Fever.}

The type of scarlet fever has now so greatly윽 altered from that prevailing 20 years ago that $a$ fatal case is rare, and the Metropolitan Asylums? Board, towards the end of last year, resolved that as scarlet fever was now such a mild ailment as은 compared with measles they had determined to $>$ give precedence to cases of measles seeking admission to their institutions. The mildness of the type of scarlet fever now prevailing may be gauged from the fact that no death has occurred in Tottenham from that cause since November, 1923. It isc incumbent on those who have the administration of health services to prepare for the contingencyo of having to treat notified cases of scarlet fevero elsewhere than in isolation hospitals. Where there? is sufficient domestic accommodation there willo be no difficulty ; indeed, that is even now the mosto satisfactory way of dealing with the prevailing? mild type of the disease.

It happens opportunely that investigationso have been proceeding apace in America, and the researches of the Dicks have resulted in theo identification of an organism, or series of organisms, 